\title{
High-dose fasudil preconditioning and postconditioning attenuate myocardial ischemia-reperfusion injury in hypercholesterolemic rats
}

\author{
NAN WU ${ }^{1}$, XIAOWEN ZHANG ${ }^{2}$ and DALIN JIA ${ }^{1}$ \\ ${ }^{1}$ Department of Cardiology, The First Affiliated Hospital of China Medical University; \\ ${ }^{2}$ Department of Medical Genetics, China Medical University, Shenyang, Liaoning 110001, P.R. China
}

Received April 24, 2013; Accepted November 15, 2013

DOI: $10.3892 / \mathrm{mmr} .2013 .1818$

\begin{abstract}
Fasudil may induce preconditioning and postconditioning against myocardial ischemia-reperfusion injury in normal rats, however, their effectivenesses in hypercholesterolemia remains to be determined. The study aimed to investigate whether fasudil induces preconditioning and postconditioning in hypercholesterolemic rats and to determine the roles of the phosphoinositol 3-kinase (PI3K)/Akt/endothelial nitric oxide synthase (eNOS) pathway and mitochondrial KATP (m-KATP) channels in this process. Isolated rat hearts underwent $30 \mathrm{~min}$ global ischemia and $120 \mathrm{~min}$ reperfusion. Low- $(1 \mathrm{mg} / \mathrm{kg})$ or high-dose $(10 \mathrm{mg} / \mathrm{kg})$ fasudil was administered $15 \mathrm{~min}$ prior to ischemia and at the initial onset of reperfusion. 5-Hydroxydecanoic acid (5HD), an m-KATP channel blocker, at $10 \mathrm{mg} / \mathrm{kg}$ was administered $5 \mathrm{~min}$ prior to reperfusion. Myocardial infarct size was estimated by 2,3,5-triphenyltetrazolium chloride (TTC) staining and lactate dehydrogenase (LDH) and creatine kinase-MB (CK-MB) were analyzed from coronary effluents. Phosphorylation of Akt and eNOS was measured by immunoblotting. High-dose fasudil-induced preconditioning and postconditioning significantly reduced infarct size and the release of LDH and CK-MB and increased the phosphorylation of Akt and eNOS compared with the control group, whereas low-dose fasudil did not exert these beneficial effects. In addition, the cardioprotection of high-dose fasudil-induced preconditioning and postconditioning are blocked by 5HD. Low-dose fasudil-induced preconditioning and postconditioning are abrogated by hypercholesterolemia, while high-dose fasudil restores the cardioprotection, which is involved in upregulation of the PI3K/Akt/eNOS pathway and inducing the opening of the m-KATP channel.
\end{abstract}

Correspondence to: Dr Dalin Jia, Department of Cardiology, The First Affiliated Hospital of China Medical University, 155th North of Nanjing Street, Heping, Shenyang, Liaoning 110001, P.R. China E-mail: jd12001@263.net

Key words: fasudil, hypercholesterolemia, preconditioning, postconditioning, mitochondrial KATP channel

\section{Introduction}

Hypercholesterolemia is one of the major risk factors for the induction and progression of ischemic heart disease. Findings of previous studies have demonstrated that hypercholesterolemia or hyperlipidemia accelerated the progression of myocardial ischemic reperfusion injury, delayed the myocardial recovery on reperfusion and deteriorated the cardioprotective effects of preconditioning and postconditioning $(1,2)$. Although a number of drugs have been shown to be effective in preventing myocardial ischemic reperfusion injury, few are capable of preserving cardioprotection in the presence of hypercholesterolemia (3).

Rho-kinase activity is involved in diverse cardiovascular diseases and regulation of Rho-kinase signaling is important for cellular function, including contraction, death, proliferation and apoptosis $(4,5)$. A previous study showed that Rho-kinase is activated in ischemic myocardium and the inhibition of Rho-kinase activity is a novel target for preventing myocardial ischemia reperfusion injury (6). Fasudil, a Rho-kinase inhibitor, not only has beneficial effects in ischemic heart disease (7), but induces preconditioning and postconditioning against myocardial ischemia reperfusion injury in normal rat $(8,9)$. However, whether fasudil is capable of inducing preconditioning and postconditioning in the hypercholesterolemic rat remains unknown. Therefore, this issue was examined in the initial phase of the study using an isolated rat model.

Inhibiting Rho-kinase may lead to activation of the phosphoinositol 3-kinase (PI3K)/Akt/endothelial nitric oxide synthase (eNOS) signal transduction pathway (10). Rho-kinase acts as a negative regulator of PI3K activation and modulates the protective effect via the PI3K/Akt/eNOS pathway on myocardial preconditioning and postconditioning (11). In addition, fasudil-induced preconditioning and postconditioning is mediated by mitochondrial KATP (m-KATP) channels in the normal rat $(9,12)$. Therefore, in the second phase of the study, fasudil was tested to determine whether it is capable of inducing preconditioning and postconditioning in the hypercholesterolemic rat and if it is involved in upregulating the PI3K/Akt/eNOS pathway and inducing the opening of the m-KATP channel.

Therefore, the present study was performed to clarify whether fasudil induces preconditioning and postconditioning 
in the hypercholesterolemic rat and if so, whether the cardioprotective effects are mediated by regulation of the PI3K/Akt/eNOS pathway and m-KATP channel.

\section{Materials and methods}

Animals. A total of 90 male Wistar rats purchased from the Center of Experimental Animals (China Medical University), weighing $200 \pm 10 \mathrm{~g}$ were used in this study. The animals used were treated in accordance with the Guide for the Care and Use of Laboratory Animals, published by the National Institutes of Health (NIH). The study instructions were approved by the Institutional Ethics Committee.

Drugs. Fasudil was purchased from Chase Sun Pharmaceutical Co., Ltd. (Tianjin, China). 5-Hydroxydecanoic acid (5HD), an m-KATP channel blocker and 2,3,5-triphenyltetrazolium chloride (TTC) were purchased from Sigma-Aldrich (St. Louis, MO, USA).

Induction of experimental hypercholesterolemia. Prior to beginning an 8-week feeding period, blood samples were collected from the rat's caudal vein for determination of plasma levels of total cholesterol (TC), high-density lipoprotein (HDL) and low-density lipoprotein (LDL) using commercial kits [Total Cholesterol Assay kit- Fluoro Cholesterol (Cell Technology Inc., Mountain View, CA, USA), Cholesterol LDL direct and Cholesterol HDL direct (both Biosystems S.A., Costa Brava, Barcelona, Spain)]. Animals were fed with diet enriched with $1.5 \%$ cholesterol, $5 \%$ egg yolk powder, $10 \%$ lard, $0.5 \%$ sodium cholate, $3 \%$ sugar and $80 \%$ normal feedstuff for 8 weeks and this formula was modified as previously reported (13). Following the 8 -week feeding period, blood samples were collected from the rat's caudal vein again for determination of serum lipid to determine the success of hypercholesterolemic models.

Heart preparation. Rats were anesthetized with an intraperitoneal injection of pentobarbital sodium $(100 \mathrm{mg} / \mathrm{kg})$. Heparin $(1,500 \mathrm{IU} / \mathrm{kg})$ was administered intravenously to prevent intracoronary clot formation. The heart was rapidly excised and immediately immersed in ice-cold heparinized modified Krebs-Henseleit (K-H) buffer containing:127 mM NaCl, $17.7 \mathrm{mM} \mathrm{NaHCO}_{3}, 5.1 \mathrm{mM} \mathrm{KCl}, 1.5 \mathrm{mMCaCl}_{2}, 1.26 \mathrm{mM} \mathrm{MgCl}_{2}$ and $11 \mathrm{mM}$ D-glucose (pH 7.4). The heart was mounted on a Langendorff perfusion apparatus and retrogradely perfused through the aorta with recirculating buffer saturated with $95 \% \mathrm{O}_{2}$ and $5 \% \mathrm{CO}_{2}$ at $37^{\circ} \mathrm{C}$. The heart was maintained in a thermostatic chamber at $37^{\circ} \mathrm{C}$. Perfusion was maintained at a constant pressure of $75 \mathrm{mmHg}$. A fluid-filled latex balloon was inserted in the left ventricle (LV) via the left atrium for pressure measurement. The balloon was connected to a pressure transducer and inflated to an initial LV end-diastolic pressure between 8 and $10 \mathrm{mmHg}$.

Experimental instructions. Rats were divided into 9 groups with 10 animals/group (Fig. 1). In all the groups, the isolated rat hearts were perfused with $\mathrm{K}-\mathrm{H}$ solution $(127 \mathrm{mM} \mathrm{NaCl}$, $17.7 \mathrm{mM} \mathrm{NaHCO}, 5.1 \mathrm{mM} \mathrm{KCl}, 1.5 \mathrm{mM} \mathrm{CaCl}_{2}, 1.26 \mathrm{mM}$ $\mathrm{MgCl}_{2}$ and $11 \mathrm{mM}$ D-glucose, $\mathrm{pH}=7.4$ ) and allowed $10 \mathrm{~min}$ stabilization. Isolated rat hearts were then subjected to $30 \mathrm{~min}$ global ischemia and 120 min reperfusion.

Isolated rat hearts for the control group were subjected to $30 \mathrm{~min}$ global ischemia and $120 \mathrm{~min}$ reperfusion. In the low-dose fasudil preconditioning group (LFpre), the isolated rat hearts were perfused with K-H solution containing $1 \mathrm{mg} / \mathrm{kg}$ dose fasudil $15 \mathrm{~min}$ prior to ischemia. In the LF postconditioning group (LFpost), the isolated rat hearts were perfused with $\mathrm{K}-\mathrm{H}$ solution containing $1 \mathrm{mg} / \mathrm{kg}$ dose fasudil at the onset of reperfusion. In the high-dose fasudil preconditioning group (HFpre), the isolated rat hearts were perfused with $\mathrm{K}-\mathrm{H}$ solution containing $10 \mathrm{mg} / \mathrm{kg}$ dose fasudil $15 \mathrm{~min}$ prior to ischemia. In the HF postconditioning group (HFpost), the isolated rat hearts were perfused with $\mathrm{K}-\mathrm{H}$ solution containing $10 \mathrm{mg} / \mathrm{kg}$ dose fasudil at the onset of reperfusion. In the LFpre + 5HD group (LFpre + 5HD), the isolated rat hearts received fasudil as described above and were administered $\mathrm{K}-\mathrm{H}$ solution containing $10 \mathrm{mg} / \mathrm{kg}$ dose $5 \mathrm{HD}$ at $5 \mathrm{~min}$ prior to reperfusion. In the LFpost $+5 \mathrm{HD}$ group (LFpost $+5 \mathrm{HD}$ ), the isolated rat hearts received fasudil and 5HD as described above. In the HFpre + 5HD group (HFpre + 5HD), the isolated rat hearts received fasudil and 5HD as described above. In the HFpost +5 HD group (HFpost +5 HD), the isolated rat hearts received fasudil and $5 \mathrm{HD}$ as described above.

Hemodynamic monitoring. The hemodynamic assessment included heart rate (HR), left ventricular developed pressure (LVDP), positive first order derivative of ventricular pressure $(+\mathrm{dp} / \mathrm{dt})$ and negative first order derivative of ventricular pressure (-dp/dt). These parameters were continuously monitored throughout the experiment. The HR, LVDP, $+\mathrm{dp} / \mathrm{dt}$ and $-\mathrm{dp} / \mathrm{dt}$ were sampled and digitally processed via a hemodynamic system (MP150; Biopac Systems, Inc., Goleta, CA, USA).

Assessment of myocardial injury. To determine the extent of myocardial injury, the release of creatine kinase-MB (CK-MB) and lactate dehydrogenase (LDH) in coronary effluents were measured using commercially available kits [Rat Creatine Kinase MB Isoenzyme (CK-MB) ELISA kit and rat LDH ELISA kit (both Bioss, Woburn, MA,USA)]. Values are expressed in international units (IU) per liter.

Measurement of infarct size. Infarct size was determined as previously described (14). Briefly, after $2 \mathrm{~h}$ reperfusion, the hearts were harvested and the left ventricles were sectioned from apex to base into 2-3-mm sections, following incubation for $20 \mathrm{~min}$ at $37^{\circ} \mathrm{C}$ in $1 \%$ TTC and unstained tissue was carefully separated from stained tissue by an independent observer. The unstained tissue represented the dead cells and the stained tissue represented the viable cells. The unstained mass was expressed as a percentage of total left ventricular mass. The total left ventricle mass also corresponds to the risk area since global ischemia was induced.

Western blotting. Following 30 min reperfusion, left ventricles were homogenized in a lysis buffer: $10 \mathrm{mM}$ Tris-HCl, $20 \mathrm{mM}$ orthophosphate, $1 \mathrm{mM}$ EGTA, $1 \mathrm{mM}$ EDTA, $2 \mathrm{mM} \mathrm{Na} \mathrm{VO}_{4}$ and $1 \mathrm{mM}$ phenylmethylsulfonyl fluoride (pH 7.4). Following sonication, the lysates were centrifuged, proteins were separated by electrophoresis on SDS-PAGE 
Table I. Biochemical analysis (mean $\pm \mathrm{SD}, \mathrm{n}=40$ for each group).

\begin{tabular}{lccc}
\hline Parameters & Total cholesterol $(\mathrm{mg} / \mathrm{dl})$ & HDL-C $(\mathrm{mg} / \mathrm{dl})$ & $\mathrm{LDL}-\mathrm{C}(\mathrm{mg} / \mathrm{dl})$ \\
\hline Prior to cholesterol-enriched diet feed & $53.7 \pm 6.1$ & $44.3 \pm 4.6$ & $14.7 \pm 4.3$ \\
Following cholesterol-enriched diet feed & $307.1 \pm 7.6^{\mathrm{a}}$ & $45.4 \pm 5.1$ & $42.5 \pm 5.3^{\mathrm{a}}$ \\
\hline
\end{tabular}

${ }^{\mathrm{a}} \mathrm{P}<0.05$ vs. prior to cholesterol-enriched diet feed. HDL-C, high density lipoprotein cholesterol; low density lipoprotein cholesterol.

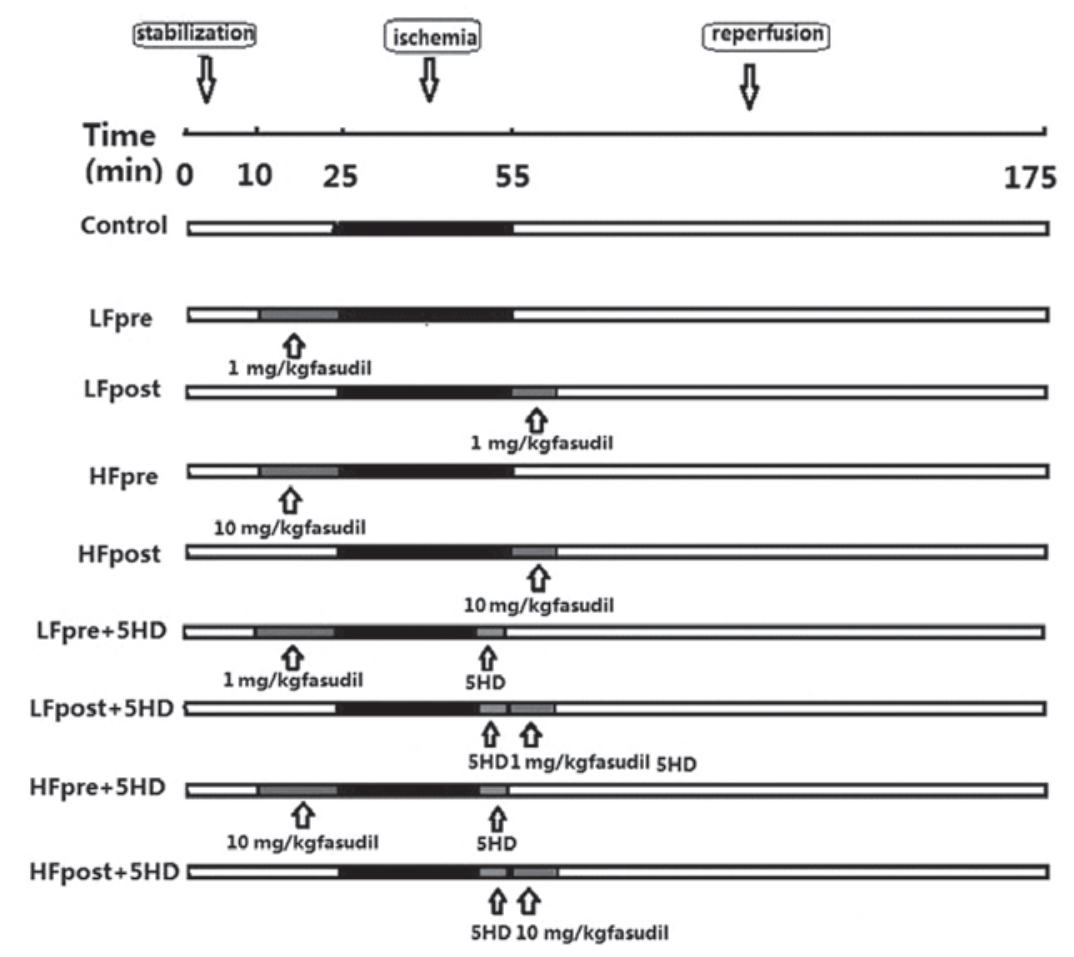

Figure 1. Representation of experimental instructions. LF, $1 \mathrm{mg} / \mathrm{kg}$ fasudil; HF, $10 \mathrm{mg} / \mathrm{kg}$ fasudil; pre, fasudil administered 15 min prior to ischemia; post, fasudil administered following onset of reperfusion; 5HD, 5-hydroxydecanoic acid. LF, low-dose fasudil; HF, high-dose fasudil.

and transferred onto polyvinylidene difluoride-plus membrane. The membranes were blocked with $5 \%$ milk, followed by incubation overnight at $4^{\circ} \mathrm{C}$ with the following antibodies: Akt (1:500); phospho-Akt (at Ser473, 1:500) eNOS (1:500) and phospho-eNOS (at Ser1177, 1:500; all Santa Cruz Biotechnology, Inc., Santa Cruz, CA, USA). Following incubation, the membranes were washed three times with $0.1 \%$ Tween-20 for $15 \mathrm{~min}$ and incubated with horseradish peroxidase (Boster, Wuhan, China) for $2 \mathrm{~h}$. Levels of phosphorylated proteins were normalized to their total protein levels. Relative densitometry was performed using a computerized software package (NIH Image 1.63 software; National Institutes of Health, Bethesda, MD, USA).

Statistical analysis. The data are expressed as mean \pm SD. Statistical analysis was performed using SigmaStat software, version 3.5 (Systat Software, Inc., Chicago, IL, USA). Differences between groups were evaluated using one-way analysis of variance (ANOVA), followed by Student-Newman-Keuls post-hoc test. $\mathrm{P}<0.05$ was considered to indicate a statistically significant difference.

\section{Results}

Plasma lipid levels. Table I shows the average values for TC, HDL and LDL in the plasma of animals prior to and following an 8-week cholesterol-enriched diet. A significant increase $(\mathrm{P}<0.05)$ in the average TC and LDL values was observed in the animals fed with the cholesterol-enriched diet as compared with the control group. There was no significant difference in the value of HDL between the two groups $(\mathrm{P}>0.05)$.

Hemodynamic changes. Table II shows the values of HR, LVDP, +dp/dt and -dp/dt at baseline and during specific times of reperfusion. No significant differences were identified within or among the groups in HR, LVDP, +dp/dt and -dp/dt at any measurement point.

Release of CK-MB and LDH measurement. Figs. 2 and 3 show the release of CK-MB and $\mathrm{LDH}$ of isolated hearts following $30 \mathrm{~min}$ global no-flow ischemia and $120 \mathrm{~min}$ reperfusion. There was no difference in the baseline values of CK-MB and LDH among all the groups $(\mathrm{P}>0.05)$. HFpre and HFpost 
Table II. HR, +dp/dt, -dp/dt and LVDP prior to and during reperfusion.

\begin{tabular}{|c|c|c|c|c|}
\hline Time & Baseline & $\mathrm{R}-10$ & $\mathrm{R}-20$ & $\mathrm{R}-60$ \\
\hline \multicolumn{5}{|l|}{ HR } \\
\hline Control & $226 \pm 23$ & $150 \pm 18$ & $174 \pm 19$ & $160 \pm 24$ \\
\hline LFpre & $217 \pm 20$ & $145 \pm 26$ & $165 \pm 23$ & $158 \pm 15$ \\
\hline LFpost & $225 \pm 17$ & $158 \pm 22$ & $178 \pm 27$ & $166 \pm 22$ \\
\hline HFpre & $220 \pm 18$ & $146 \pm 17$ & $176 \pm 12$ & $164 \pm 16$ \\
\hline HFpost & $215 \pm 16$ & $143 \pm 21$ & $168 \pm 15$ & $165 \pm 12$ \\
\hline LFpre + 5HD & $227 \pm 21$ & $141 \pm 26$ & $159 \pm 23$ & $149 \pm 25$ \\
\hline LFpost + 5HD & $221 \pm 22$ & $139 \pm 22$ & $172 \pm 22$ & $166 \pm 17$ \\
\hline HFpre + 5HD & $230 \pm 18$ & $136 \pm 27$ & $164 \pm 22$ & $148 \pm 26$ \\
\hline HFpost + 5HD & $212 \pm 26$ & $140 \pm 18$ & $170 \pm 15$ & $155 \pm 24$ \\
\hline \multicolumn{5}{|l|}{ LVDP (mmHg) } \\
\hline Control & $86 \pm 6.4$ & $34 \pm 5.4$ & $38 \pm 5.1$ & $34 \pm 3.2$ \\
\hline LFpre & $84 \pm 6.5$ & $32 \pm 7.3$ & $38 \pm 4.5$ & $33 \pm 4.3$ \\
\hline LFpost & $83 \pm 6.6$ & $36 \pm 7.9$ & $37 \pm 6.0$ & $32 \pm 3.4$ \\
\hline HFpre & $88 \pm 7.5$ & $33 \pm 8.4$ & $34 \pm 7.7$ & $30 \pm 4.9$ \\
\hline HFpost & $84 \pm 6.9$ & $34 \pm 6.7$ & $39 \pm 4.2$ & $34 \pm 3.4$ \\
\hline LFpre + 5HD & $86 \pm 4.4$ & $34 \pm 5.4$ & $35 \pm 5.1$ & $32 \pm 3.8$ \\
\hline LFpost + 5HD & $85 \pm 7.3$ & $32 \pm 7.7$ & $37 \pm 6.5$ & $31 \pm 6.3$ \\
\hline HFpre + 5HD & $89 \pm 5.6$ & $35 \pm 7.2$ & $36 \pm 6.6$ & $40 \pm 7.4$ \\
\hline HFpost + 5HD & $87 \pm 8.5$ & $31 \pm 9.4$ & $34 \pm 5.4$ & $36 \pm 4.9$ \\
\hline \multicolumn{5}{|l|}{$+\mathrm{dp} / \mathrm{dt}$} \\
\hline Control & $2,322 \pm 233$ & $1,589 \pm 103$ & $1,622 \pm 208$ & $1,362 \pm 198$ \\
\hline LFpre & $2,318 \pm 187$ & $1,466 \pm 259$ & $1,589 \pm 217$ & $1,284 \pm 134$ \\
\hline LFpost & $2,472 \pm 274$ & $1,546 \pm 202$ & $1,666 \pm 244$ & $1,354 \pm 143$ \\
\hline HFpre & $2,312 \pm 233$ & $1,589 \pm 103$ & $1,602 \pm 221$ & $1,362 \pm 198$ \\
\hline HFpost & $2,298 \pm 177$ & $1,466 \pm 259$ & $1,589 \pm 217$ & $1,284 \pm 114$ \\
\hline LFpre + 5HD & $2,402 \pm 244$ & $1,536 \pm 207$ & $1,566 \pm 287$ & $1,454 \pm 203$ \\
\hline LFpost + 5HD & $2,231 \pm 262$ & $1,489 \pm 301$ & $1,684 \pm 212$ & $1,335 \pm 158$ \\
\hline HFpre + 5HD & $2,331 \pm 251$ & $1,519 \pm 301$ & $1,584 \pm 231$ & $1,315 \pm 138$ \\
\hline HFpost + 5HD & $2,330 \pm 198$ & $1,476 \pm 225$ & $1,601 \pm 201$ & $1,378 \pm 167$ \\
\hline \multicolumn{5}{|l|}{$-\mathrm{dp} / \mathrm{dt}$} \\
\hline Control & $1,476 \pm 222$ & $1,178 \pm 178$ & $1,422 \pm 117$ & $1,238 \pm 156$ \\
\hline LFpre & $1,378 \pm 207$ & $1,109 \pm 167$ & $1,271 \pm 189$ & $1,079 \pm 134$ \\
\hline LFpost & $1,408 \pm 190$ & $1,189 \pm 312$ & $1,308 \pm 202$ & $1,136 \pm 234$ \\
\hline HFpre & $1,400 \pm 156$ & $1,146 \pm 242$ & $1,298 \pm 345$ & $1,178 \pm 198$ \\
\hline HFpost & $1,476 \pm 222$ & $1,158 \pm 168$ & $1,406 \pm 177$ & $1,202 \pm 176$ \\
\hline LFpre + 5HD & $1,395 \pm 137$ & $1,129 \pm 187$ & $1,271 \pm 189$ & $1,169 \pm 144$ \\
\hline LFpost + 5HD & $1,428 \pm 180$ & $1,109 \pm 232$ & $1,408 \pm 206$ & $1,106 \pm 153$ \\
\hline HFpre + 5HD & $1,410 \pm 176$ & $1,176 \pm 232$ & $1,278 \pm 275$ & $1,078 \pm 208$ \\
\hline HFpost + 5HD & $1,401 \pm 165$ & $1,098 \pm 198$ & $1,274 \pm 237$ & $1,156 \pm 159$ \\
\hline
\end{tabular}

Data are presented as means $\pm \mathrm{SD}$ and $\mathrm{n}=7$ for each. $\mathrm{LF}, 1 \mathrm{mg} / \mathrm{kg}$ fasudil; $\mathrm{HF}, 10 \mathrm{mg} / \mathrm{kg}$ fasudil; pre, fasudil administered $15 \mathrm{~min}$ prior to ischemia; post, fasudil administered following onset of reperfusion; 5HD, 5-hydroxydecanoic acid. HR, heart rate; +dp/dt, positive first order derivative of ventricular pressure; -dp/dt, negative first order derivative of ventricular pressure; LVDP, left ventricular developed pressure; LF, low-dose fasudil; HF, high-dose fasudil; LFpre, LF preconditioning, LFpost; LF, postconditioning; HFpre, HF, preconditioning; HFpost, HF, postconditioning.

significantly reduced the release of CK-MB and LDH in hypercholesterolemic rat hearts as compared with the control group $(\mathrm{P}<0.05)$. However, LFpre and LFpost did not decrease the release of $\mathrm{CK}-\mathrm{MB}$ and $\mathrm{LDH}$ in hypercholesterolemic rat hearts $(\mathrm{P}>0.05)$. In addition, 5HD treatment failed to significantly attenuate the release of CK-MB and LDH by high-dose 


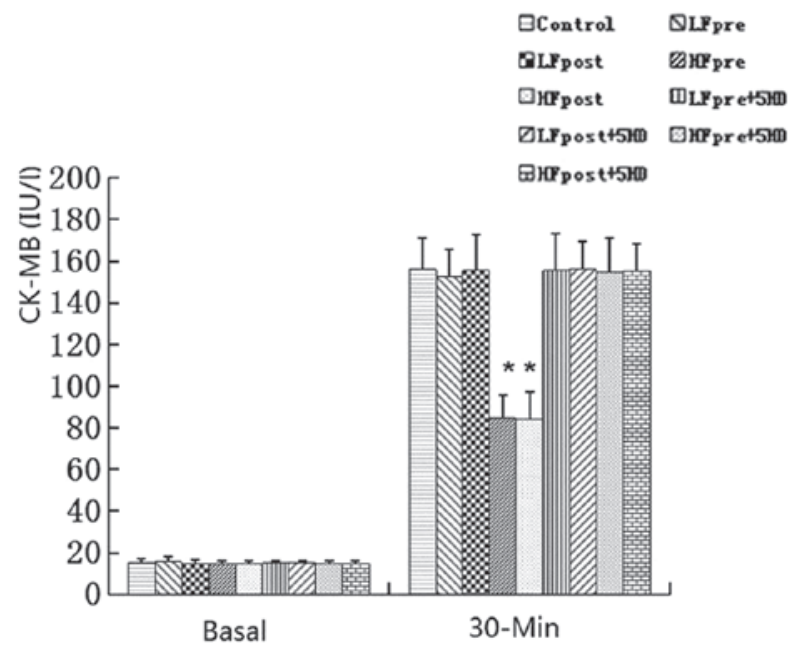

Figure 2. Release of CK-MB in isolated rat heart. LF, $1 \mathrm{mg} / \mathrm{kg}$ fasudil; $\mathrm{HF}, 10 \mathrm{mg} / \mathrm{kg}$ fasudil; pre, fasudil administered $15 \mathrm{~min}$ prior to ischemia; post, fasudil administered following the onset of reperfusion. Data are presented as means $\pm \mathrm{SD}$ and $\mathrm{n}=6$ for each. ${ }^{*} \mathrm{P}<0.05$ vs. control. CK-MB, creatine kinase-MB; LF, low-dose fasudil; HF, high-dose fasudil; 5HD, 5-hydroxydecanoic acid.

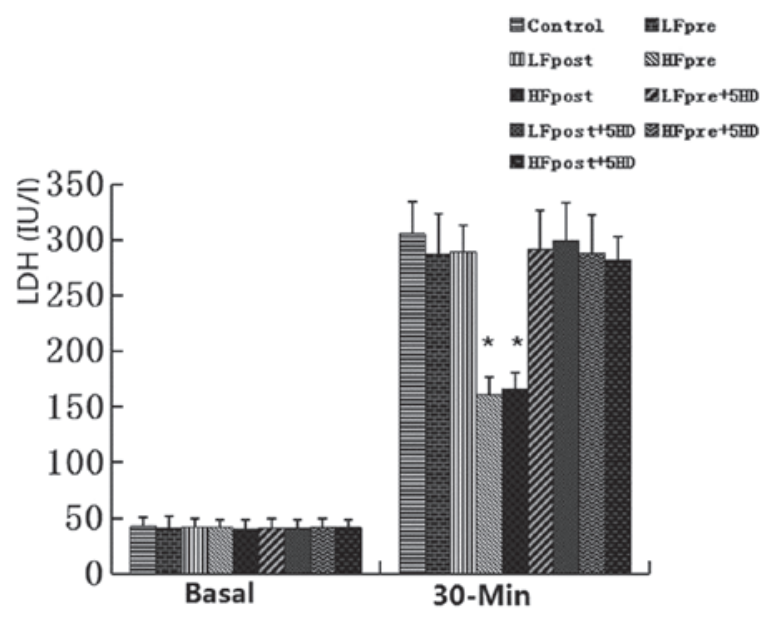

Figure 3. Release of LDH in isolated rat heart. LF, $1 \mathrm{mg} / \mathrm{kg}$ fasudil; HF, $10 \mathrm{mg} / \mathrm{kg}$ fasudil; pre, fasudil administered $15 \mathrm{~min}$ prior to ischemia; post, fasudil administered following the onset of reperfusion. Data are presented as means $\pm \mathrm{SD}$ and $\mathrm{n}=6$ for each. ${ }^{*} \mathrm{P}<0.05$ vs. control. $\mathrm{LDH}$, lactate dehydrogenase; HF, high-dose fasudil; 5HD, 5-hydroxydecanoic acid.

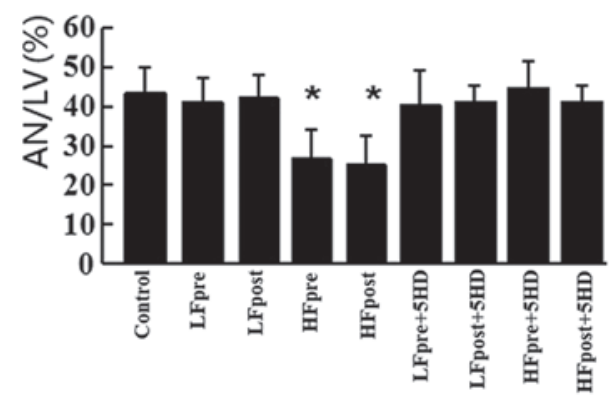

Figure 4. AN expressed as a percentage of LV area. LF, $1 \mathrm{mg} / \mathrm{kg}$ fasudil; $\mathrm{HF}, 10 \mathrm{mg} / \mathrm{kg}$ fasudil; pre, fasudil administered $15 \mathrm{~min}$ prior to ischemia; post, fasudil administered following onset of reperfusion. Data are presented as means $\pm \mathrm{SD}$ and $\mathrm{n}=6$ for each. ${ }^{*} \mathrm{P}<0.05$ vs. control. AN Area of necrosis; LV, left ventrical; LF, low-dose fasudil; HF, high-dose fasudil; 5HD, 5-hydroxydecanoic acid.

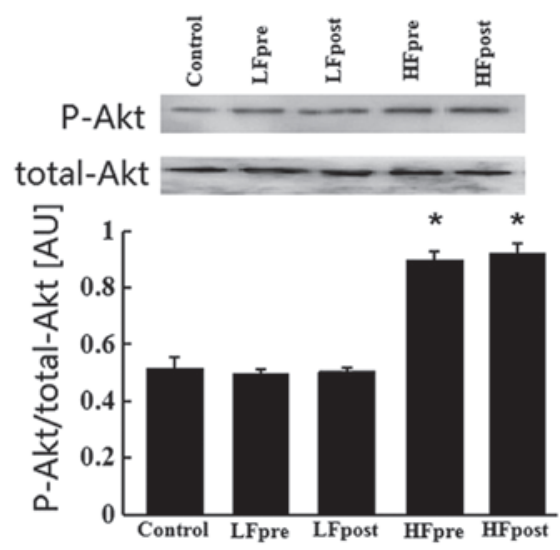

Figure 5. Myocardial total Akt and p-Akt expression. LF, $1 \mathrm{mg} / \mathrm{kg}$ fasudil; $\mathrm{HF}, 10 \mathrm{mg} / \mathrm{kg}$ fasudil; pre, fasudil administered $15 \mathrm{~min}$ prior to ischemia; post, fasudil administered following onset of reperfusion. Data are presented as means \pm SD and $n=3$ for each. " $\mathrm{P}<0.05$ vs. control. LF, low-dose fasudil; HF, high-dose fasudil.

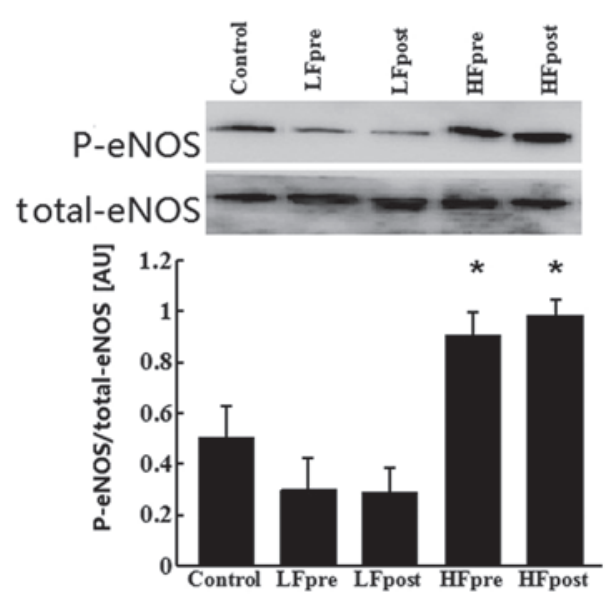

Figure 6. Myocardial total eNOS and p-eNOS expression. LF, $1 \mathrm{mg} / \mathrm{kg}$ fasudil; $\mathrm{HF}, 10 \mathrm{mg} / \mathrm{kg}$ fasudil; pre, fasudil administered $15 \mathrm{~min}$ prior to ischemia; post, fasudil administered following onset of reperfusion. Data are presented as means $\pm \mathrm{SD}$ and $\mathrm{n}=3$ for each. ${ }^{*} \mathrm{P}<0.05$ vs. control. $\mathrm{LF}$, low-dose fasudil; HF, high-dose fasudil.

fasudil-induced preconditioning and postconditioning in hypercholesterolemic rat hearts as compared with the control group $(\mathrm{P}>0.05)$.

Infarct size measurement. As shown in Fig. 4, infarct size was measured as an area of necrosis expressed as a percentage of the left ventricular area. Infarct size was significantly reduced by HFpre and HFpost as compared with the control group $(26.9 \pm 6.9,25.3 \pm 7.3$ vs. $43.6 \pm 6.2 \%$; $\mathrm{P}<0.05)$. However, LFpre and LFpost did not reduce infarct size as compared with the control group $(41.3 \pm 5.8,42.3 \pm 5.6$ vs. $43.6 \pm 6.2 \% ; \mathrm{P}>0.05)$ and there was no significant difference between the HFpre and HFpost groups (26.9 \pm 6.9 vs. $25.3 \pm 7.2 \%$; $\mathrm{P}>0.05$ ). In addition, HFpre and HFpost failed to significantly reduce infarct size when treated with 5HD as compared with the control group (44.7 $\pm 6.6,41.1 \pm 4.3$ vs. $43.6 \pm 6.2 \%$; $P>0.05$ ).

Expression of Akt and eNOS proteins. As shown in Figs. 5 and 6, Akt phosphorylation and eNOS phosphorylation were 
enhanced in the HFpre and HFpost groups as compared with the control group $(0.899 \pm 0.030,0.921 \pm 0.034$ vs. $0.516 \pm 0.037$; $\mathrm{P}<0.05)$, but no significant alteration in Akt and eNOS phosphorylation was observed between these two groups $(0.899 \pm 0.030$ vs. $0.921 \pm 0.034 ; \mathrm{P}>0.05)$. In addition, there was no significant change in Akt and eNOS phosphorylation in the LFpre and LFpost groups as compared with the control group $(0.497 \pm 0.013,0.505 \pm 0.012$ vs. $0.516 \pm 0.037 ; \mathrm{P}>0.05)$.

\section{Discussion}

Previously, a number of studies have demonstrated that fasudil induces the pharmacological preconditioning and postconditioning in normal rat $(8,9)$. The present study shows that high-dose fasudil preserves preconditioning and postconditioning against myocardial ischemia reperfusion injury under hypercholesterolemia in rats and is involved in upregulating the PI3K/Akt/eNOS pathway and inducing the opening of the m-KATP channel.

Controversies remains in the literature with regard to improvement in postischemic functional recovery of fasudil preconditioning. Nishizawa et al (15) observed that fasudil preconditioning did not improve recovery of the myocardium following ischemic reperfusion and even prevented KATP channel-induced improvement in postischemic functional recovery. By contrast, Demiryürek et al (8) showed that fasudil, at 1 and $10 \mathrm{mg} / \mathrm{kg}$ doses, caused significant decreases in the mean arterial blood pressure. However, no significant change in mean arterial blood pressure was observed with fasudil at $0.3 \mathrm{mg} / \mathrm{kg}$ dose (8). In the current study, fasudil preconditioning at 1 or $10 \mathrm{mg} / \mathrm{kg}$ doses was observed to improve the functional recovery of the myocardium following ischemic reperfusion in hypercholesterolemic rats. In addition, fasudil postconditioning at 1 and $10 \mathrm{mg} / \mathrm{kg}$ doses did not improve cardiac function recovery in the presence of hypercholesterolemia. A similar result was reported by Ichinomiya et al (9), which showed that 0.15 and $0.5 \mathrm{mg} / \mathrm{kg}$ doses of fasudil postconditioning did not improve cardiac function recovery under hyperglycemia, as well as normoglycemia.

Although fasudil induces the pharmacological preconditioning and postconditioning in the normal rat, whether fasudil is capable of inducing preconditioning and postconditioning in hypercholesterolemic rat remains unknown. The present study showed that high-dose fasudil preconditioning and postconditioning markedly decreased myocardial infarct size and reduced the release of CK-MB and LDH in hypercholesterolemic rat hearts as compared with the control group. However, low-dose fasudil preconditioning and postconditioning failed to decrease myocardial infarct size and reduced the release of CK-MB and LDH as compared with the control group. It is hypothesized that hypercholesterolemia blocked low-dose fasudil-induced preconditioning, while postconditioning and high-dose fasudil restores the cardioprotection. Ma et al $(16,17)$ reported that hypercholesterolemia induced by a cholesterol-rich diet may increase the myocardial Rho-kinase activity, thus, hypercholesterolemia was hypothesized to increase the myocardial Rho-kinase activity during ischemia-reperfusion and raise the threshold of fasudil-induced preconditioning and postconditioning. In addition, Demiryürek et al (8) firstly demonstrated that cardioprotection of fasudil had a dose-dependent effect. Only $10 \mathrm{mg} / \mathrm{kg}$ dose fasudil was capable of mimicking the beneficial effects of ischemic and pharmacological preconditioning in anesthetized rats (8). Ichinomiya et al (9) also showed that only high-dose fasudil preserved postconditioning against myocardial infarction under hyperglycemia in rats. The present study also demonstrated that cardioprotection of fasudil preconditioning and postconditiong tended to exhibit a dose-dependent effect in the hypercholesterolemic rat.

Preconditioning and postconditioning have been shown to involve the activation of the PI3K/Akt/eNOS pathway and ultimately prevent the opening of the mitochondrial permeability transition pore (18). Wolfrum et al (10) showed that the cardioprotective effect of Rho-kinase inhibition prior to ischemia is involved in PI3K/Akt and nitric oxide synthase (NOS) activation. Hamid et al (6) showed that inhibition of Rho-kinase by Y27632, a structurally unrelated inhibitor of fasudil, at reperfusion onset limited infarct size through an Akt/eNOS-dependent mechanism. In addition, a number of types of pharmacological preconditioning and postconditioning were shown to depend on the PI3K/Akt/eNOS pathway $(19,20)$. The present western blot analysis revealed that high-dose fasudil preconditioning and postconditioning markedly increased the phosphorylation of Akt and eNOS as compared with the control group, but low-dose fasudil preconditioning and postconditioning did not increase the phosphorylation of Akt and eNOS as compared with the control group. The results confirm a previous report that hypercholesterolemia blocked phosphorylation of Akt and eNOS in postconditioned myocardium (21). It was also observed that high-dose fasudil preserved preconditioning and postconditioning against myocardial ischemia-reperfusion in hypercholesterolemic rats involved in upregulating the PI3K/Akt/eNOS pathway.

The m-KATP channel is a target downstream of the $\mathrm{PI} 3 \mathrm{~K} / \mathrm{Akt} / \mathrm{eNOS}$ pathway and an effector in cardioprotection by ischemic preconditioning and postconditioning $(22,23)$. Fasudil-induced preconditioning and postconditioning is dependent on activation of the m-KATP channel $(9,12)$. The current study reported that the preconditioning and postconditioning stimulus of fasudil was eliminated by $5 \mathrm{HD}$, an m-KATP channel blocker and suggested that it is likely that fasudil induced preconditioning and postconditioning in hypercholesterolemic rats is mediated by m-KATP channels.

In conclusion, low-dose fasudil-induced preconditioning postconditioning are abrogated by hypercholesterolemia, but high-dose fasudil restores the cardioprotection, which is involved in upregulating the PI3K/Akt/eNOS pathway and inducing the opening of the m-KATP channel.

\section{Acknowledgements}

This study was supported by a grant from the Liaoning Provincial Science and Technology Projects, China (no. 2009225051-1).

\section{References}

1. Iliodromitis EK, Zoga A, Vrettou A, et al: The effectiveness of postconditioning and preconditioning on infarct size in hypercholesterolemic and normal anesthetized rabbits. Atherosclerosis 188: 356-362, 2006. 
2. Ferdinandy P, Schulz R and Baxter GF: Interaction of cardiovascular risk factors with myocardial ischemia/reperfusion injury, preconditioning, and postconditioning. Pharmacol Rev 59: 418-458, 2007.

3. Balakumar P and Babbar L: Preconditioning the hyperlipidemic myocardium: fact or fantasy? Cell Signal 24: 589-595, 2012.

4. Nunes KP, Rigsby CS and Webb RC: RhoA/Rho-kinase and vascular diseases: what is the link? Cell Mol Life Sci 67: 3823-3836, 2010.

5. Satoh K, Fukumoto Y and Shimokawa H: Rho-kinase: important new therapeutic target in cardiovascular diseases. Am J Physiol Heart Circ Physiol 301: H287-H296, 2011.

6. Hamid SA, Bower HS and Baxter GF: Rho-kinase activation plays a major role as a mediator of irreversible injury in reperfused myocardium. Am J Physiol Heart Circ Physiol 292: H2598-H2606, 2007.

7. Fukumoto Y, Mohri M, Inokuchi K, et al: Anti-ischemic effects of fasudil, a specific Rho-kinase inhibitor, in patients with stable effort angina. J Cardiovasc Pharmacol 49: 117-121, 2007.

8. Demiryürek S, Kara AF, Celik A, Babül A, Tarakçioglu M and Demiry ürek AT: Effects of fasudil, a Rho-kinase inhibitor, on myocardial preconditioning in anesthetized rats. Eur J Pharmacol 527: 129-140, 2005.

9. Ichinomiya T, Cho S, Higashijima U, Matsumoto S, Maekawa T and Sumikawa K: High-dose fasudil preserves postconditioning against myocardial infarction under hyperglycemia in rats: role of mitochondrial KATP channels. Cardiovasc Diabetol 11: 28, 2012.

10. Wolfrum S, Dendorfer A, Rikitake Y, et al: Inhibition of Rhokinase leads to rapid activation of phosphatidylinositol 3-kinase/protein kinase Akt and cardiovascular protection Arterioscler Thromb Vasc Biol 24: 1842-1847, 2004

11. Manintveld OC, Verdouw PD and Duncker DJ: The RISK of ROCK. Am J Physiol Heart Circ Physiol 292: H2563-H2565, 2007.

12. Zhao JL, Yang YJ, Pei WD, Sun YH, You SJ and Gao RL: Remote periconditioning reduces myocardial no-reflow by the activation of K ATP channel via inhibition of Rho-kinase. Int J Cardiol 133: 179-184, 2009.

13. Zhao H, Wang Y, Wu Y, et al: Hyperlipidemia does not prevent the cardioprotection by postconditioning against myocardial ischemia/reperfusion injury and the involvement of hypoxia inducible factor-1alpha upregulation. Acta Biochim Biophys Sin (Shanghai) 41: 745-753, 2009.
14. Jia D: The protective effect of mitochondrial ATP-sensitive $\mathrm{K}^{+}$ channel opener, nicorandil, combined with $\mathrm{Na}^{+} / \mathrm{Ca}^{2+}$ exchange blocker KB-R7943 on myocardial ischemia-reperfusion injury in rat. Cell Biochem Biophys 60: 219-224, 2011

15. Nishizawa K, Wolkowicz PE, Yamagishi T, Guo LL and Pike MM: Fasudil prevents KATP channel-induced improvement in postischemic functional recovery. Am J Physiol Heart Circ Physiol 288: H3011-H3015, 2005.

16. Ma Z, Zhang J, Du R, Ji E and Chu L: Rho kinase inhibition by fasudil has anti-inflammatory effects in hypercholesterolemic rats. Biol Pharm Bull 34: 1684-1689, 2011.

17. Ma Z,Zhang J, Ji E, Cao G, Li G and Chu L: Rho kinase inhibition by fasudil exerts antioxidant effects in hypercholesterolemic rats. Clin Exp Pharmacol Physiol 38: 688-694, 2011.

18. Hausenloy DJ, Tsang A and Yellon DM: The reperfusion injury salvage kinase pathway: a common target for both ischemic preconditioning and postconditioning. Trends Cardiovasc Med 15: 69-75, 2005.

19. Sumi S, Kobayashi H, Yasuda S, Iwasa M, Yamaki T, Yamada Y, et al: Postconditioning effect of granulocyte colony-stimulating factor is mediated through activation of risk pathway and opening of the mitochondrial KATP channels. Am J Physiol Heart Circ Physiol 299: H1174-H1182, 2010.

20. Hönisch A, Theuring N, Ebner B, Wagner C, Strasser RH and Weinbrenner C: Postconditioning with levosimendan reduces the infarct size involving the PI3K pathway and KATP-channel activation but is independent of PDE-III inhibition. Basic Res Cardiol 105: 155-167, 2010.

21. Andreadou I, Farmakis D, Prokovas E, et al: Short-term statin administration in hypercholesterolaemic rabbits resistant to postconditioning: effects on infarct size, endothelial nitric oxide synthase, and nitro-oxidative stress. Cardiovasc Res 94: 501-509, 2012.

22. Ghosh S, Standen NB and Galiñanes M: Evidence for mitochondrial K ATP channels as effectors of human myocardial preconditioning. Cardiovasc Res 45: 934-940, 2000.

23. Mykytenko J, Reeves JG, Kin H, et al: Persistent beneficial effect of postconditioning against infarct size: role of mitochondrial $\mathrm{K}(\mathrm{ATP})$ channels during reperfusion. Basic Res Cardiol 103: 472-484, 2008. 